\title{
Preparation and characterization of Au nanoparticles for theranostic applications
}

\section{Ines Delfino ${ }^{1}$, Maria Lepore ${ }^{2, *}$, Rosarita Tatè ${ }^{3}$ and Marianna Portaccio ${ }^{2}$}

1 Dipartimento di Scienze Ecologiche e Biologiche, Università della Tuscia, Viterbo, ITALY; EMail: delfino@unitus.it (I.D.)

2 Dipartimento di Medicina Sperimentale, Seconda Università di Napoli, Napoli, ITALY; E-Mails: maria.lepore@unina2.it (M.L.); marianna.portaccio@unina2.it (M.P.)

3 Institute of Genetics and Biophysics - ABT, Napoli, ITALY; E-Mail: rosarita.tate@igb.cnr.it (R.T.)

* Author to whom correspondence should be addressed; E-Mail: maria.lepore@unina2.it (M.L.); Tel.: +39-081-5665839; Fax: +39-081-5667500.

Published: 1 June 2014

\begin{abstract}
Gold nanoparticles (GNPs) are very attractive materials due to their unique properties of small size, large surface area to volume ratio, high reactivity to the living cells, stability over high temperatures. These properties along with the evidence that GNPs are amenable to the attachment of biomolecules or ligands have led to the realization of several nanoparticle-based bio-devices for many theranostic applications and still boost a huge research effort in the field. In the present work absorption spectroscopy, static and dynamic light scattering, Fourier-Transform infrared (FT-IR) microspectroscopy and TEM microscopy were used to characterize different sized bare and biotinylated GNPs (20-70 $\mathrm{nm}$ diameter range). The aggregation process of the biotinylated particles induced by addition of neutravidin in the nanomolar range was investigated as a proof of concept of the approach. In particular, optical visible techniques were used for estimating the size of particles before and after the biotin capping procedure, FT-IR microspectroscopy and TEM microscopy to investigate the biochemical morphological changes occurring in GNPs after interaction processes.
\end{abstract}

Keywords: bare and biotinylated gold nanopaticles; static light scattering; dynamic light scattering; Fourier-Transform infrared (FT-IR) microspectroscopy; TEM microscopy 


\section{Introduction}

Nanotechnology and nanoscience, interdisciplinary subjects of physics, chemistry, biology, and engineering, advanced the contemporary fundamental and application research in the past two decades. Among many nanomaterials, gold nanoparticles (GNPs) and their colloidal dispersions have attracted great interest due to their unique properties of small size, large surface area to volume ratio, high reactivity to the living cells, stability over high temperatures [1,2]. These properties along with the evidence that GNPs are amenable to the attachment of biomolecules or ligands through well-known thiol and amino chemistry or simply by electrostatic interactions have led to a wealth of nanoparticlebased bio-devices for many theranostic applications and the research effort in the field is still huge. The characterization of the properties of eventual new bio-nanomaterials is one important topic of the present research effort [3,4]. In the present work absorption spectroscopy, static and dynamic light scattering, Fourier-Transform infrared (FT-IR) microspectroscopy and TEM microscopy have been used to characterize different sized bare and biotinylated GNPs (from 20 to $70 \mathrm{~nm}$ diameter). These experimental techniques have been also applied to investigate the aggregation process of the biotinylated particles induced by addition of neutravidin at various concentrations in the nanomolar range. In particular, optical visible techniques have been used for estimating the size of particles before and after the biotin capping procedure. FT-IR microspectroscopy has allowed us to investigate the biochemical changes occurring in GNPs after interaction processes while TEM microscopy has enabled to observe the relative morphological modifications. The complete characterization of these processes is of fundamental importance for further manipulations required for GNPs theranostic applications [5].

\section{Experimental Section}

\subsection{Materials}

Hydrogen tetrachloroaurate $\left(\mathrm{HAuCl}_{4}\right)$, trisodium citrate, neutravidin, and biotinamidocaproyl labeled bovine serum albumin (biotinylated BSA, BBSA) were purchased from Sigma-Aldrich (Sigma-Aldrich Co., USA). All chemicals were used as received.

\subsection{Nanoparticle preparation}

GNPs were obtained by conventional citrate reduction method [6]. A $0.01 \% \mathrm{HAuCl}_{4}$ solution was reduced by $1 \%$ sodium citrate with vigorous stirring at near boiling temperature. GNP size was controlled by adjusting the amounts of sodium citrate. Solutions at various concentrations of GNPs with an expected diameter in the $20-70 \mathrm{~nm}$ were prepared (B, C, D, E, F and G GNP preparations).

The surface modification of $20 \mathrm{~nm}$ gold nanoparticles was performed using the procedure described in Refs. [7,8]. Briefly, a proper amount of the GNP solution was mixed with aqueous solution of biotinylated BSA (to obtain $[\mathrm{BBSA}]=1.44 \mathrm{mg} / \mathrm{ml}$ ), and this mixture was incubated at room temperature for $2 \mathrm{~h}$. The GNP/BBSA mixture was then centrifuged in an Eppendorf centrifuge tube equipped with a $100000 \mathrm{MW}$ cutoff filter for $10 \mathrm{~min}$, using an Eppendorf microcentrifuge at $8000 \mathrm{~g}$, to separate the BBSA-coated GNPs (BBSA-GNPs) from the excess BBSA. The supernatant was carefully 
removed, and the pellet containing the BBSA-GNP was resuspended in $10 \mathrm{mM}$ sodium phosphate buffer ( $\mathrm{pH}$ 7). B preparation of GNPs was employed for capping and aggregation experiments.

\subsection{Sample characterization methods}

Absorption spectra of the prepared Au nanoparticles and their conjugates with biomolecules were recorded by UV-vis spectroscopy at a resolution of $1.0 \mathrm{~nm}$ on Perkin Elmer LS25. All spectra were collected over the range of 200-800 nm with $2.0 \mathrm{~nm}$ resolution. Transmission electron microscope (TEM) images were obtained by a JEOL JEM-1011 TEM (JEOL, Tokyo, Japan) equipped with a thermionic tungsten filament and operated at an acceleration voltage of $100 \mathrm{kV}$. Images were taken using a Morada cooled slow-scan CCD camera (3783X2672 pixels) and micrographs were taken with iTEM software (Olympus Soft Imaging System GmbH, Munster, Germany). Particle sizing by DLS and $\zeta$-Potential measurements were performed using a Zetasizer Nano Series and Dynamic Light Scattering (Malvern Instruments Ltd., Malvern, UK). Fourier Transform InfraRed (FT-IR) spectra of the samples were recorded by using a Perkin Elmer Spectrum One FT-IR spectrometer equipped with a MultiScope FT-IR Infrared Microscope and a Mercury Cadmium Telluride detector (MCT) to record micro-ATR (for further details see Refs. [9,10]).

Absorption spectroscopy was used to study the stability of the solutions and to consequently choose the GNP concentration for the best stability, to evaluate the diameter of the naked GNP [11] along with the completion of the BBSA conjugation to GNPs and its effects on GNPs optical properties [7]. TEM and DLS measurements were used to estimate the size of GNPs and BBSA-GNPs. $\zeta$-Potential measurements were used to evaluate the characteristics of the surface of naked GNPs while by FT-IR spectroscopy the BBSA after the interaction with GNPs was studied.

\subsection{Aggregation assays}

The aggregation of BBSA-GNPs as a consequence of addition of neutravidin at various concentrations was studied by Static Light Scattering (SLS), DLS and Resonance Light Scattering (RLS) as described in details in Ref. [8]. For SLS and DLS, the above described DLS system was used. RLS spectra were collected in the $250-800 \mathrm{~nm}$ range at room temperature by a Perkin-Elmer, model LS55, equipped with $450 \mathrm{~W}$ Xenon lamp and two monochromators. The spectrofluorometer was used in synchroscan mode with the same excitation and emission wavelength $(\Delta \lambda=0)$. The bandpass width for the excitation and emission monochromators was $2.0 \mathrm{~nm}$. An integration time of $1.0 \mathrm{~s}$, that relates to $0.5 \mathrm{~nm}$ step, was used. The light emitted by each sample kept in a $1.0 \mathrm{~cm} \times 1.0 \mathrm{~cm}$ internal size quartz cuvette was collected at right angle to the excitation radiation. RLS spectra were corrected for variations in lamp intensity.

\section{Results and Discussion}

The GNP solutions feature different optical absorption, as expected, that results in a difference in the perceived color of the solutions themselves. In Figure 1 the image of the cuvettes (Fig. 1a) filled with $\mathrm{B}, \mathrm{C}$ and D preparations is shown along with the corresponding absorption spectra (Fig. 1b), for 
which the position of the maximum is labeled. The same is shown in Figure 2 for $E, F$ and $G$ preparations.

Figure 1. (a) Image of cuvettes containing B, C and D preparations. (b) Absorption spectra of $\mathrm{B}, \mathrm{C}$ and $\mathrm{D}$ preparations.
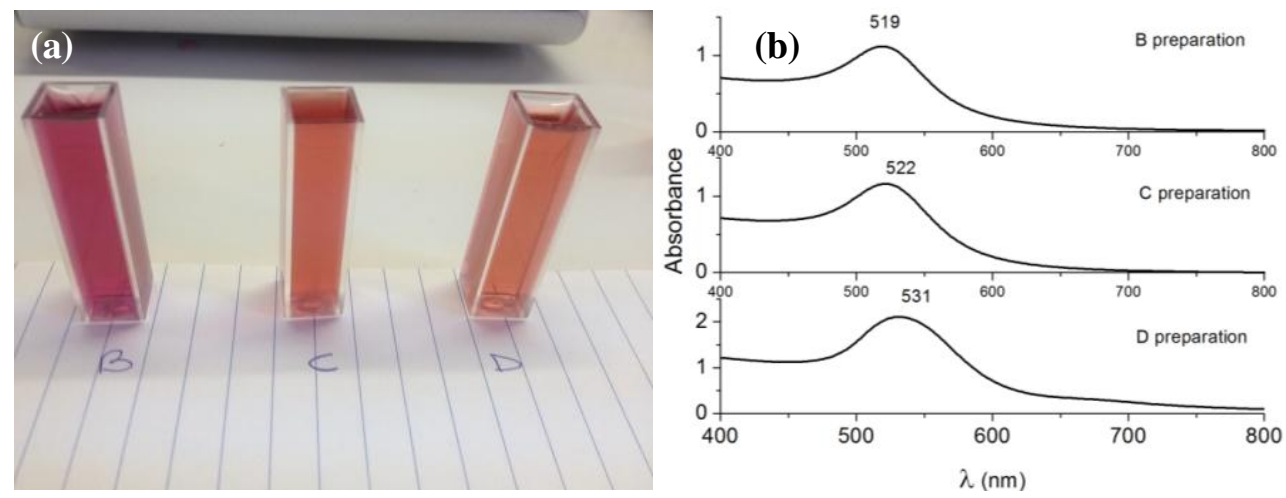

Figure 2. (a) Image of cuvettes containing E, F and G preparations. (b) Absorption spectra of $\mathrm{E}, \mathrm{F}$ and $\mathrm{G}$ preparations.
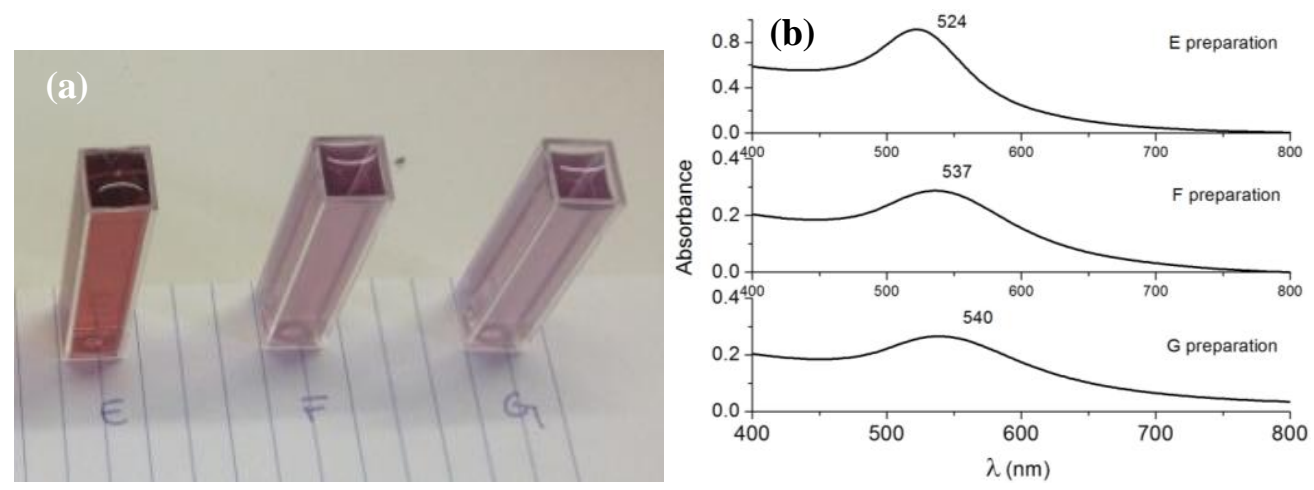

The observed spectra are characterized by the presence of a single broad peak in the 400-800 nm range, as expected [11]. In fact, it represents the surface plasmon resonance (SPR) absorption band of the metal nanoparticles which can provide valuable information on the size, structure and aggregation of GNPs. Accordingly, from the position of plasmon peak the mean diameter of the GNP of each preparation has been estimated [11]. Values of $50 \pm 5,20 \pm 2,27 \pm 3,32 \pm 3,62 \pm 6$, and $68 \pm 7 \mathrm{~nm}$ have been obtained for B, C, D, E, F and G GNP preparation, respectively. The stability of the preparations have been studied by detecting the absorption spectra for several days and the SPR peak position has been extracted. The results suggest that the preparations named B, C and D are stable for more than 6 months. These preparations have been further investigated by TEM. Representative TEM images, reported in Figure 3, and their analysis have confirmed the spherical geometry of GNPs and the size obtained by studying SPR absorption even if a bigger dispersion is suggested. GNPs from the B and D preparations have been selected to be capped with BBSA following the above described procedure. Before this procedure began, GNPs from B preparation have been further characterized by means of DLS and $\zeta$-Potential. This measurements have provided a mean diameter of $18 \pm 10 \mathrm{~nm}$ and a $\zeta-$ Potential value of $24 \pm 2 \mathrm{mV}$. BBSA-GNPs have been then studied by VIS absorption and FT-IR and 
Figure 3. TEM images of (a) B, (b) C, and (c) D preparation. Scale bar $=100 \mathrm{~nm}$.
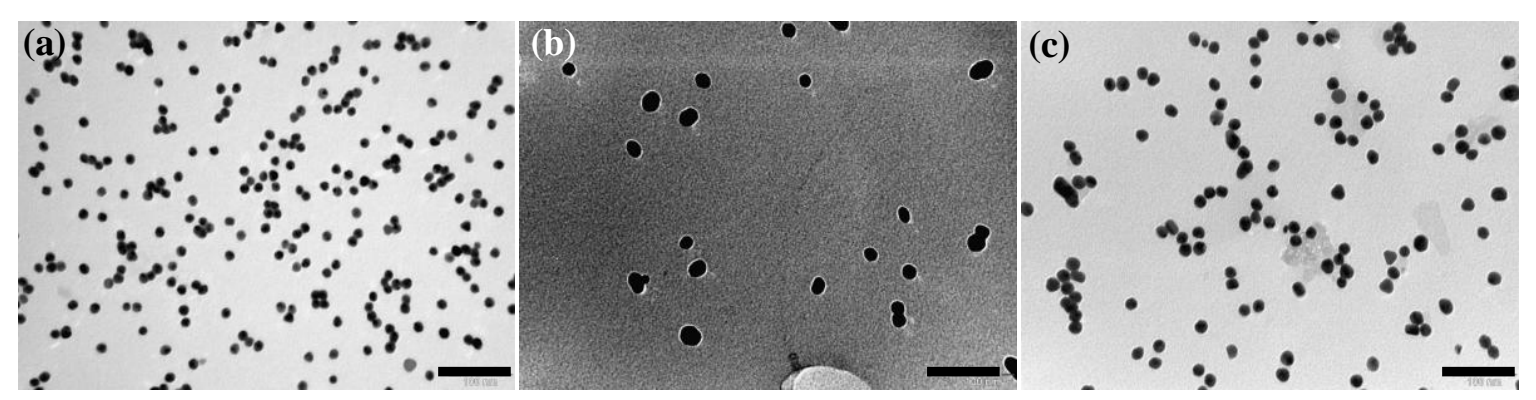

by TEM. Absorption spectra of BBSA-GNP solutions at various BBSA concentrations, shown in Figure 4 for GNPs from B preparation, demonstrate that BBSA concentration greatly affects the maximum absorbance value. TEM investigation confirms the formation of a layer on GNP surface, as witnessed by representative images reported in Figure 5. FT-IR spectroscopy further confirms the presence of BBSA in the sample (data not shown).

BBSA-GNP solutions have been used in the aggregation assays described in Ref. [8] by exploiting the characteristics of a well defined and well-known model system, i.e., biotin and one of its partners, neutravidin [7]. Neutravidin in the nanomolar concentration range has been added to BBSA-GNP solutions and RLS, DLS and SLS measurements have been performed. Results similar to those reported in literature $[5,8]$ have been obtained thus proving the efficacy of the employed procedure for GNP preparation and characterization.

Figure 4. Absorption spectra of BBSA-GNP solutions obtained using B preparation at different BBSA concentrations.

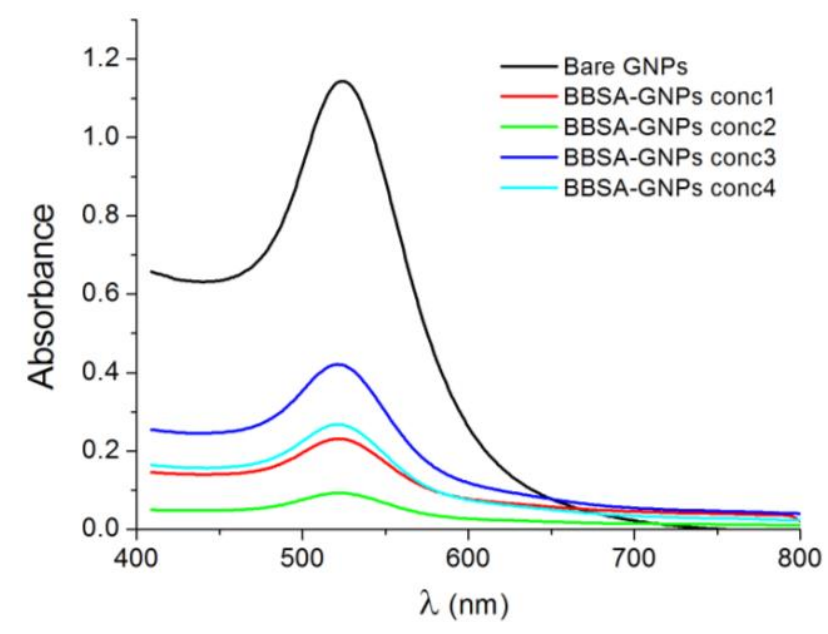

\section{Conclusions/Outlook}

The results of absorption spectroscopy, static and dynamic light scattering, Fourier-Transform infrared (FT-IR) microspectroscopy and TEM microscopy measurements used for characterizing different sized bare and biotinylated GNPs (20-70 $\mathrm{nm}$ diameter range) have been reported. They have shown the usefulness of this multitechnique approach for nanoparticles characterization and aggregation study. This is of great importance since the complete characterization of GNPs is of fundamental importance for further manipulations required for theranostic applications. 
Figure 5. (a) TEM image of BBSA-GNPs from B preparation. Scale bar $=200 \mathrm{~nm}$. (b) TEM image of BBSA-GNPs from D preparation. Scale bar $=100 \mathrm{~nm}$.

(a)

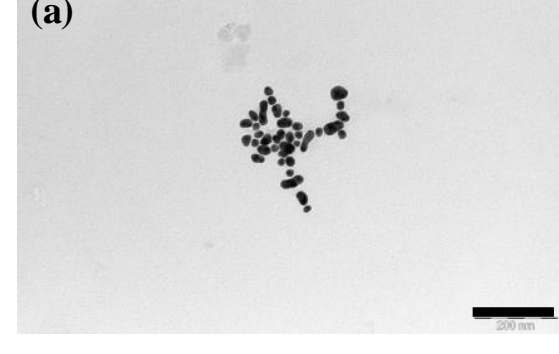

(b)

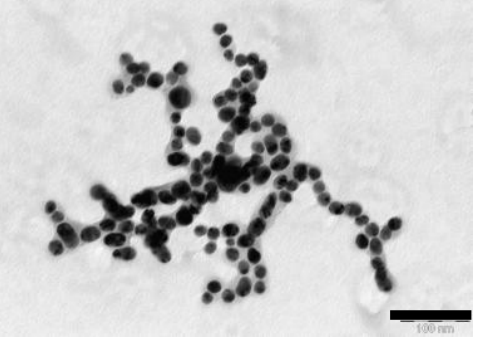

\section{References}

1. G.F. Paciotti, G.F.; Myer, L.; Weinreich, D.; Goia, D.; Pavel, N.; McLaughlin, R.E.; Tamarkin, L. Colloidal gold: a novel nanoparticle vector for tumor directed drug delivery. Drug Deliv. 2004, 11, 169-183.

2. Paciotti, G.F.; Kingston, D.G.I.; Tamarkin, L. Colloidal gold nanoparticles: a novel nanoparticle platform for developing multifunctional tumor-targeted drug delivery vectors. Drug Dev. Res. 2006, 67, 47-54.

3. Ghosh, P.; Han, G.; De, M.; Kim, C.K.; Rotello, V.M. Gold nanoparticles in delivery applications. Adv. Drug Deliv. Rev. 2008, 60, 1307-1315.

4. Davis, M. E; Chen, Z.; Shin, D. M. Nanoparticle therapeutics: an emerging treatment modality for cancer. Nature Rev. Drug Discovery 2008, 7, 771-782.

5. Heo, D.N.; Yang, D.H.; Moon, H.J.; Lee, J.; Bae, M.S.; Lee, S.C.; Lee, W.J.; Sun, I.C.; Kwon, K. Gold nanoparticles surface-functionalized with paclitaxel drug and biotin receptor as theranostic agents for cancer therapy. Biomaterials 2012, 33, 856e866.

6. Frens, G. Particle size and sol stability in metal colloids. Colloid Polym. Sci. 1972, 250, 736-741.

7. Aslan, K.; Holley, P.; Davies, L.; Lakowicz, J.R.; Geddes, C.D. Angular-Ratiometric Plasmon Resonance Light Scattering for Bioaffinity Sensing, J. Am. Chem. Soc. 2005, 127, 12115-12121.

8. Delfino, I. Light Scattering Methods for Tracking Gold Nanoparticles Aggregation Induced by Biotin-Neutravidin Interaction. Biophys. Chem. 2013, 177-178, 7-13.

9. Delfino, I; Portaccio, M.; Della Ventura, B.; Mita, D.G.; Lepore, M. Enzyme distribution and secondary structure of sol-gel immobilized glucose oxidase by micro attenuated total reflection FT-IR spectroscopy. Mat. Sci. Engin. C 2013, 33, 304-310.

10. Portaccio, M.; Della Ventura, B.; Mita, D.G.; Manolova, N.; Stoilova, O.; Rashkov, I.; Lepore, M. FT-IR Microscopy Characterization of sol-gel layers prior and after glucose oxidase immobilization for biosensing applications. J. Sol-Gel Sci. Technol. 2011, 57, 204-211.

11. Haiss, W.; Thanh, N.T.K.; Aveyard, J.; Ferni, D.G. Determination of Size and Concentration of Gold Nanoparticles from UV-Vis Spectra. Anal. Chem. 2007, 79, 4215-4221.

(C) 2014 by the authors; licensee MDPI, Basel, Switzerland. This article is an open access article distributed under the terms and conditions of the Creative Commons Attribution license (http://creativecommons.org/licenses/by/3.0/). 